(2) Open Access Full Text Article

\title{
Investigation of prostate resected weight on postoperative sexual dysfunction following transurethral resection of prostate surgery: a population-based study
}

This article was published in the following Dove Medical Press journal:

Therapeutics and Clinical Risk Management

\author{
Fu-Chao Liu' ${ }^{1,2, *}$ \\ Shih-Jyun Shen ${ }^{1,2, *}$ \\ Jr-Rung $\operatorname{Lin}^{1-3}$ \\ Huang-Ping Yu ${ }^{1,2,4}$ \\ 'Department of Anesthesiology, \\ Chang Gung Memorial Hospital, \\ Taoyuan, Taiwan; ${ }^{2}$ College of Medicine, \\ Chang Gung University, Taoyuan, \\ Taiwan; ${ }^{3}$ Clinical Informatics and \\ Medical Statistics Research Center \\ and Graduate Institute of Clinical \\ Medicine, Chang Gung University, \\ Taoyuan, Taiwan; ${ }^{4}$ Department of \\ Anesthesiology, Xiamen Chang Gung \\ Hospital, Xiamen, China \\ *These authors contributed equally \\ to this work
}

\begin{abstract}
Background: Benign prostate hyperplasia, a common disease in elderly men, can be surgically treated with transurethral resection of the prostate (TURP). Postoperative sexual dysfunction is a major issue and is influenced by many factors. The present study aimed to assess whether the intraoperative resected prostate weight influences the probability of postoperative sexual dysfunction.
\end{abstract}

Methods: This population-based study included 41,574 patients from the Nation Health Insurance Research Database who had undergone TURP once between 1997 and 2013. All patients were divided into three groups according to the resected prostate weight (low, medium, and high groups). Perioperative risk factors influencing sexual function were analyzed. The chi-squared test and Fisher's exact test were used to analyze differences in demographic data. The Cox proportional hazard regression analysis was used to analyze the HRs. All statistical analyses were two-sided, and a $P$-value $<0.05$ was considered statistically significant.

Results: Of the 41,574 patients, 1,168 had postoperative sexual dysfunction after surgery. The incidence was not significantly different among the three resected prostate weight groups. Younger patients and patients with histories of chronic renal disease, ischemic heart disease, and obesity had higher prevalence of postoperative sexual dysfunction. Additionally, the onset time of sexual dysfunction was not significantly different among the three resected prostate weight groups.

Conclusion: Among patients undergoing TURP in Taiwan, the resected prostate weight does not seem to be related to the presence or onset time of postoperative sexual dysfunction.

Keywords: benign prostate hyperplasia, new-onset postoperative sexual dysfunction, population-based study

\section{Introduction}

Benign prostate hyperplasia (BPH), a common disease in aging men, ${ }^{1,2}$ is treated with drug administration or surgical intervention. The disease itself or the treatment approach might cause erectile dysfunction (ED). Some researchers reported that drug administration or surgical intervention improves symptoms of lower urinary tract syndrome as well as ED. ${ }^{3-5}$ Additionally, Li et al reported that $14 \%$ of all patients who received surgical intervention had some resolution of ED symptoms; however, the authors also reported that $16 \%$ of patients experienced worsening symptoms postoperatively. ${ }^{6}$
Correspondence: Huang-Ping Yu Department of Anesthesiology, Chang Gung Memorial Hospital, 5 Fu-Shin Street, Kwei-Shan, Taoyuan 333, Taiwan Tel $+8863328 I 200$ ext 2324 Fax +88633281200 ext 2787 Email yuhp200I@adm.cgmh.org.tw 
On the other hand, another study indicated that nerve injuryrelated electroporation heat may result in postoperative ED. ${ }^{7}$

When a patient is diagnosed with $\mathrm{BPH}$ and surgery is planned, the urologist generally arranges for transurethral resection of the prostate (TURP) to improve symptoms because of its good outcomes. ${ }^{8}$ Preoperatively, the weight of the prostate part to be resected is measured and assessed with ultrasound. Theoretically, the larger the weight of the prostate part to be removed, the higher the risk of ED-related nerve damage. Recent studies of the association between TURP and postoperative ED focused on different surgical intervention methods, the difference between drug administration and surgical intervention, and the effects of bipolar and unipolar electroporation. ${ }^{6,9-11}$ However, few studies have examined the relation between the resected prostate weight and postoperative, new-onset ED. Hence, the present study aimed to assess whether the intraoperative resected prostate weight influences the probability of postoperative sexual dysfunction in patients undergoing TURP.

\section{Methods}

\section{Data collection}

This retrospective study used the National Health Insurance Research Database (NHIRD), which was developed by Taiwan's National Health Insurance systems. This database includes registration data of Taiwanese patients in hospital and outpatient departments, including diagnostic codes of the International Classification of Diseases, Revision 9, and Clinical Modification (ICD-9-CM). We searched the NHIRD and enrolled patients who underwent TURP.

This study was evaluated and approved by the NHIRD research committee and the institutional review board of Chang Gung Medical Foundation (104-6697B). The Bureau of National Health Insurance has collected claim data in a de-identified and computerized format and established the NHIRD. The data accessed is anonymized.

\section{Patient selection}

We searched the NHIRD between 1997 and 2013 and identified patients who underwent TURP once, using the ICD-9-CM codes 79406B (TURP with resected prostate weight between 5 and $15 \mathrm{~g}$ ), 79411B (TURP with resected prostate weight between 15 and $50 \mathrm{~g}$ ), and 79412B (TURP with resected prostate weight $>50 \mathrm{~g}$ ). The exclusion criteria were age $<40$ or $\geq 80$ years, death during hospitalization, and preoperative sexual dysfunction. A patient history of conditions that might influence the incidence of sexual dysfunction was assessed using ICD-9-CM codes (chronic kidney disease [ICD-9-CM 585], diabetes mellitus [ICD-9-CM 250], hypertension [ICD-9-CM 401-405], ischemic heart disease [ICD-9-CM 410-414], and obesity [ICD-9-CM 278]). Sexual dysfunction was identified by ICD-9-CM codes 302 and 607. Sexual dysfunction occurring within 1 year after TURP was associated with the surgical intervention.

\section{Outcome}

The primary outcome was the relationship between the intraoperative, resected prostate weight and the incidence of postoperative, new-onset sexual dysfunction. The secondary outcome was the relationship between the intraoperative resected prostate weight and the time of postoperative new-onset sexual dysfunction.

\section{Statistical analysis}

The chi-squared test and Fisher's exact test were used to analyze differences in demographic data. The Cox proportional hazard regression analysis was used to analyze the HRs. All statistical analyses were two-sided, and a $P$-value $<0.05$ was considered statistically significant. All analyses were performed using SPSS software.

\section{Results}

We identified 56,359 patients who underwent TURP once between 1997 and 2013. We excluded 10,543 patients who were aged $<40$ or $\geq 80$ years, 100 patients who died during hospitalization, and 4,142 patients who were diagnosed with sexual dysfunction preoperatively. The remaining 41,574 patients were included in this study. We divided the patients into three groups based on the intraoperatively resected prostate weight: low prostate weight group (resected prostate weight between 5 and $15 \mathrm{~g} ; \mathrm{N}=15,964$ ), medium prostate weight group (resected prostate weight between 15 and $50 \mathrm{~g} ; \mathrm{N}=21,861$ ), and high prostate weight group (resected prostate weight $>50 \mathrm{~g}$; $\mathrm{N}=3,749$ ). The study flow diagram is shown in Figure 1.

We analyzed the distribution of intraoperative resected prostate weight among different age groups (Figure 2) and found that the number of TURP surgery patients and the intraoperatively resected prostate weight increased with age.

Among all patients, the mean patient age was 69.38 years, and the median and IQR of the onset time of postoperative sexual dysfunction for the three groups was 1.33 (2.48), 1.24 (2.37), and 1.49 (2.62) years, respectively. We assessed the factors that might cause sexual problems (Table 1). The proportions of these factors were as follows: chronic renal failure, 3.07\%; diabetes mellitus, 20.72\%; hypertension, $53.00 \%$; ischemic heart disease, $24.36 \%$; and obesity, $0.27 \%$. 


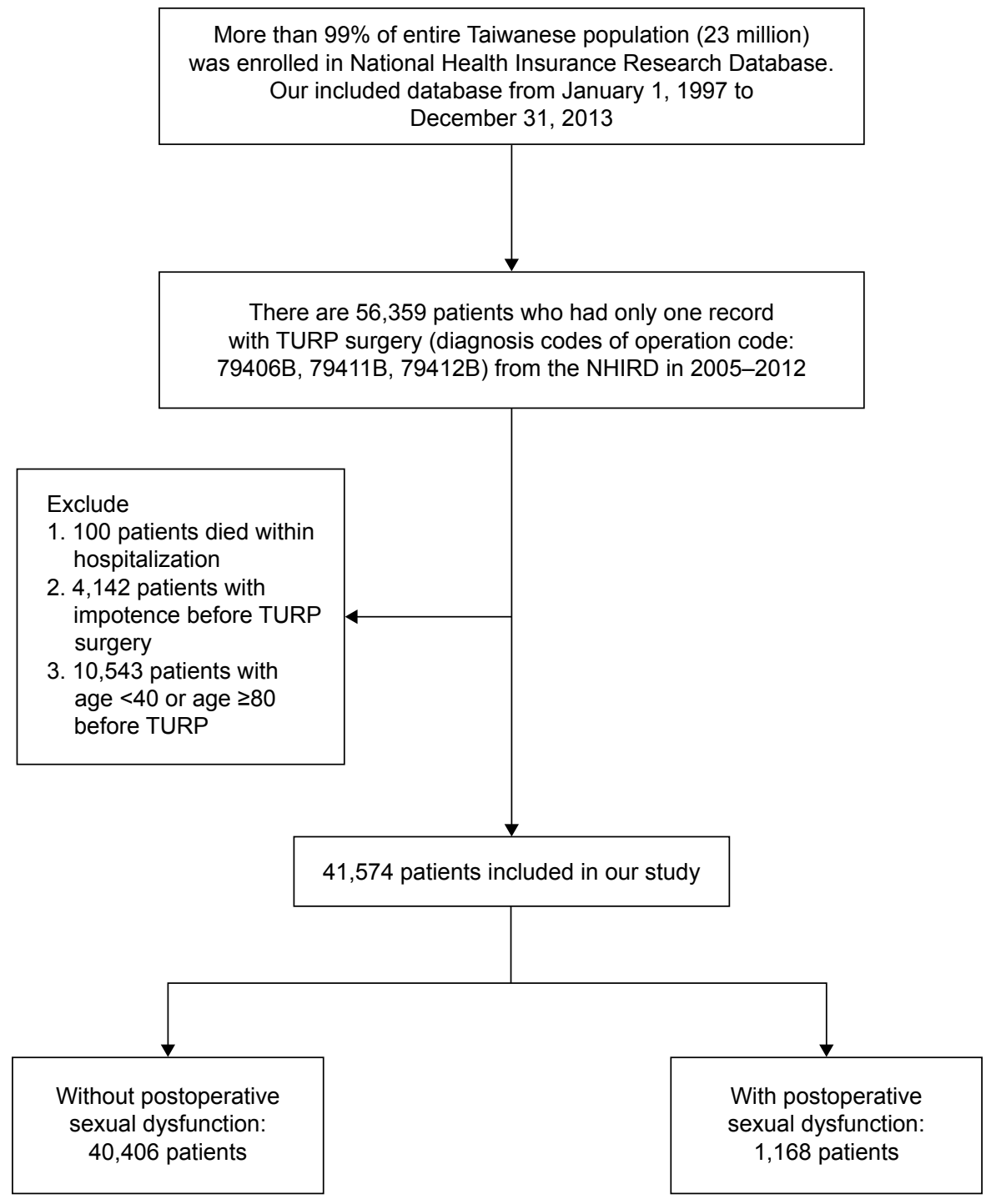

Figure I Flow diagram of the study.

Abbreviation: TURP, transurethral resection of the prostate.

Of the 41,574 patients, 1,168 had sexual dysfunction after TURP, while 40,406 patients did not. We compared relevant factors between these two patient groups and found no significant differences in all factors, except young age $(P<0.01)$, chronic renal failure $(P<0.01)$, ischemic heart disease $(P<0.01)$, and obesity $(P<0.01)$ (Table 2$)$. Patients with histories of these conditions had a high prevalence of postoperative sexual dysfunction.

In order to eliminate the impact of relevant factors, Cox proportional hazard regression was used to adjust for age and other pre-TURP diseases. Data are presented in Table 3. The risk was not significantly different among the low, medium (HR: $0.981,95 \%$ CI: $0.869,1.108, P=0.76$ ), and high prostate weight groups (HR: $0.028,95 \% \mathrm{CI}: 0.749,1.151, P=0.50$ ). These results indicated no influence on postoperative sexual dysfunction after adjustment. Thus, young patients and patients with histories of chronic renal failure, ischemic heart disease, and obesity had a higher rate of sexual dysfunction after TURP, and there was no relationship between the intraoperative resected prostate weight and postoperative sexual dysfunction.

We evaluated 1,168 patients with postoperative sexual dysfunction and analyzed the association between the intraoperative resected prostate weight and the onset time of sexual dysfunction (Table 4). The onset time of sexual dysfunction was not significantly different among the three resected prostate weight groups. Additionally, the occurrence of sexual dysfunction was not significantly different among the three groups at any of the time points assessed (6 weeks, 3, 6, 12, 24, 48 months; $P=0.29,0.74,0.94,0.57$, $0.53,0.84$, respectively). 


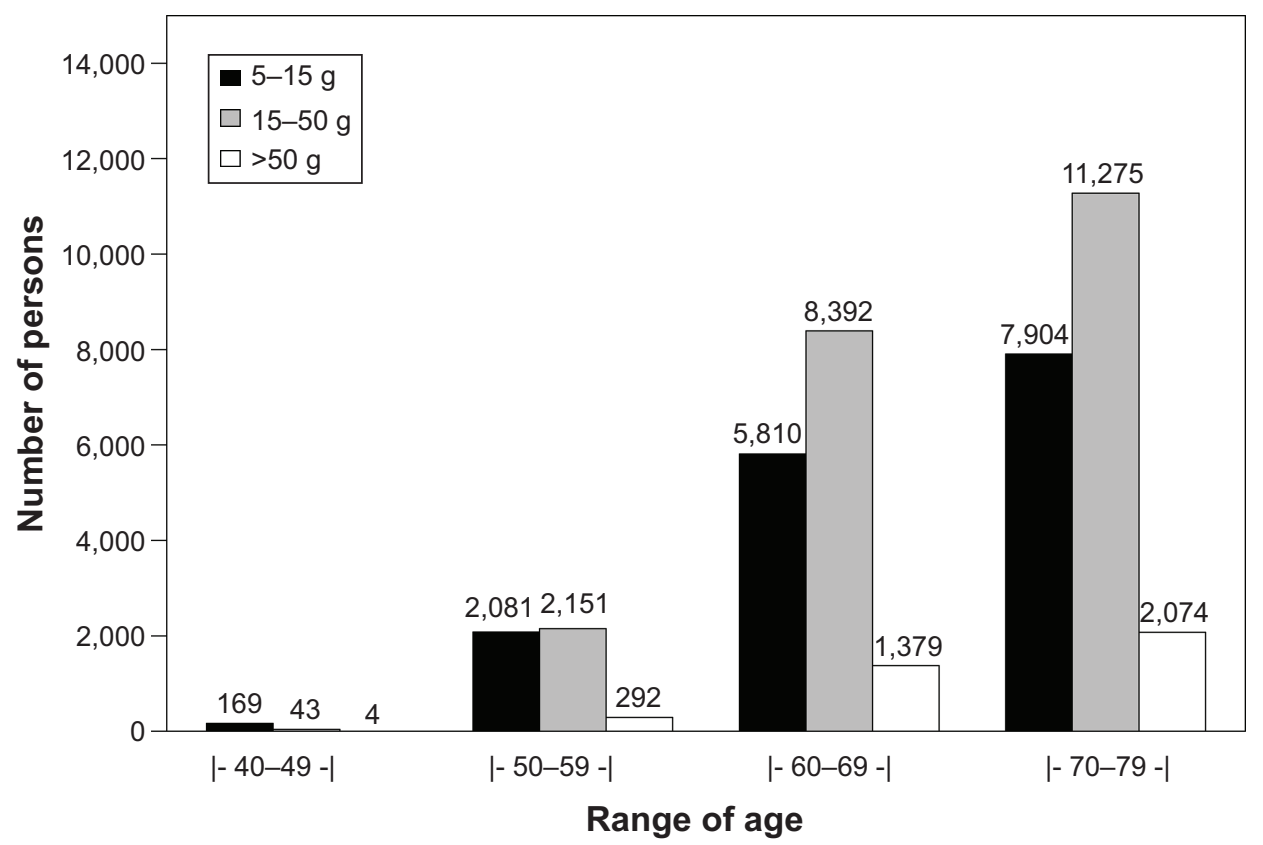

Figure 2 The distribution of patients' age and intraoperative resected weight of prostate.

\section{Discussion}

BPH and sexual dysfunction are highly prevalent diseases in elderly patients. Several theories have attempted to explain the relationship among these conditions, including decreased nitric oxide levels and nitric oxide synthesis, autonomic hyperactivity, metabolic syndrome effect, upregulation of Rho kinase in the penis, ${ }^{12}$ and pelvic atherosclerosis. ${ }^{13}$ In patients with lower urinary tract syndrome and an American Urological Association (AUA) score $<8$ points, medication is suggested initially. Some studies have mentioned that first-line medications such as $\alpha$-blockers, $5 \alpha$-reductase inhibitors, and phosphodiesterase inhibitors might relieve sexual dysfunction symptoms. ${ }^{14}$ On the other hand, surgical intervention is

Table I General demographics and preoperative diseases

\begin{tabular}{l|l}
\hline Age & $69.38(7.02)$ \\
Mean (SD) & \\
Resected prostate weight during TURP surgery $(\mathrm{g})$ & \\
$\quad 5-15$ & $15,964(38.40 \%)$ \\
I5-50 & $21,861(52.58 \%)$ \\
$>50$ & $3,749(9.02 \%)$ \\
Chronic renal failure & $1,276(3.07)$ \\
Diabetes mellitus & $8,616(20.72 \%)$ \\
Hypertension & $22,036(53.00 \%)$ \\
Ischemic heart disease & $10,129(24.36 \%)$ \\
Obesity & $111(0.27 \%)$ \\
\hline
\end{tabular}

Note: Data are presented as mean \pm SD or number of patients (\%).

Abbreviation: TURP, transurethral resection of the prostate. recommended when the AUA score is $>10$ or when the prostate is $30-80 \mathrm{~mL} .{ }^{15}$

In BPH patients, different types of surgical procedure, such as bipolar and unipolar TURP approaches, have been performed. ${ }^{11}$ Zong et al compared postoperative sexual dysfunction among traditional TURP, transurethral electrovaporization of the prostate, and holmium laser enucleation of the prostate and found that transurethral electrovaporization of the prostate was associated with a higher rate of postoperative ED. ${ }^{16}$

Table 2 Risk factor and postoperative sexual dysfunction

\begin{tabular}{|c|c|c|c|}
\hline \multirow[t]{2}{*}{ Risk factor } & \multicolumn{2}{|c|}{$\begin{array}{l}\text { Postoperative sexual } \\
\text { dysfunction }\end{array}$} & \multirow[t]{2}{*}{$P$-value } \\
\hline & No $(N=40,406)$ & Yes $(N=I, 168)$ & \\
\hline $\begin{array}{l}\text { Resected prostate } \\
\text { weight during TURP } \\
\text { surgery }(\mathrm{g})\end{array}$ & & & 0.94 \\
\hline $5-15$ & $|5,5| 4(38.40)$ & $450(38.53)$ & \\
\hline $15-50$ & $21,245(52.58)$ & $616(52.74)$ & \\
\hline$>50$ & $3,647(9.03)$ & $102(8.73)$ & \\
\hline Age & $69.47(7.00)$ & $66.08(7.03)$ & $<0.01$ \\
\hline Chronic renal failure & $1,256(3.11)$ & 20 (I.7I) & $<0.01$ \\
\hline Diabetes mellitus & $8,379(20.74)$ & $237(20.29)$ & 0.71 \\
\hline Hypertension & $21,405(52.97)$ & $631(54.02)$ & 0.48 \\
\hline Ischemic heart disease & $9,803(24.26)$ & $326(27.91)$ & $<0.01$ \\
\hline Obesity & $101(0.25)$ & $10(0.86)$ & $<0.01$ \\
\hline
\end{tabular}

Note: Data are presented as mean \pm SD or number of patients (\%). Abbreviation: TURP, transurethral resection of the prostate. 
Table 3 Cox model

\begin{tabular}{|c|c|c|c|c|}
\hline & \multicolumn{2}{|l|}{ Univariate analysis } & \multicolumn{2}{|c|}{ Multivariate analysis } \\
\hline & HR $(95 \% \mathrm{Cl})$ & $P$-value & HR (95\% Cl) & $P$-value \\
\hline \multicolumn{5}{|l|}{ TURP size (g) } \\
\hline $5-15$ & - & - & - & - \\
\hline $15-50$ & $0.98 \mathrm{I}(0.869, \mathrm{I} .108)$ & 0.76 & $1.069(0.946,1.208)$ & 0.29 \\
\hline$>50$ & $0.928(0.749,1.15 \mathrm{I})$ & 0.50 & I.073 (0.865, I.332) & 0.52 \\
\hline Age & $0.94 I(0.934,0.948)$ & $<0.001$ & $0.938(0.930,0.945)$ & $<0.01$ \\
\hline Chronic renal failure & $0.588(0.378,0.914)$ & 0.02 & $0.638(0.409,0.993)$ & 0.05 \\
\hline Ischemic heart disease & 1.231 (I.083, I.398) & $<0.01$ & I.5II (I.326, I.722) & $<0.01$ \\
\hline Obesity & $3.635(1.951,6.774)$ & $<0.001$ & $3.194(1.713,5.956)$ & $<0.01$ \\
\hline
\end{tabular}

Abbreviation: TURP, transurethral resection of the prostate.

Surgical intervention might cause thermal injury leading to erectile nerve injury, resulting in ED. ${ }^{7}$ A higher resected prostate weight was considered to be associated with a higher chance of nerve injury. However, we found no significant association between high resected prostate weight and postoperative sexual dysfunction. This result might have been obtained because we analyzed the absolute resected prostate weight, not the relative resected prostate weight compared to the total prostate weight. Tscholl et al reported that a smaller resected prostate size was associated with a lower incidence of postoperative sexual dysfunction and that higher age was associated with a higher incidence of postoperative sexual problems. ${ }^{17}$ To our knowledge, no previous population-based study has assessed whether the resected prostate weight was associated with postoperative sexual dysfunction. A previous report found that the risk of ED was higher in patients aged $>65$ years than in those aged $<65$ years. ${ }^{18}$ However, our study showed that the risk of postoperative sexual dysfunction was higher in younger patients than in older patients, though the reason is unclear. In addition, our study showed that sexual dysfunction did not occur early even when the resected prostate weight was high.

This population-based cohort study had some limitations. First, we extracted NHIRD data using ICD-9-CM codes. However, there might have been some coding mistakes due

Table 4 Cumulative rate of postoperative sexual dysfunction

\begin{tabular}{l|l|l|l|l}
\hline Time & $\begin{array}{l}\mathbf{5}-\mathbf{I 5} \mathbf{g} \\
\mathbf{( N = 1 5 , 9 6 4 )}\end{array}$ & $\begin{array}{l}\text { I5-50 g } \\
\mathbf{( N = 2 1 , 8 6 I )}\end{array}$ & $\begin{array}{l}>\mathbf{5 0} \mathbf{g} \\
\mathbf{( N = 3 , 7 4 9 )}\end{array}$ & P-value \\
\hline 6 weeks & $26(0.16)$ & $25(0.1 \mathrm{I})$ & $3(0.08)$ & 0.29 \\
3 months & $65(0.4 I)$ & $87(0.40)$ & $12(0.32)$ & 0.74 \\
6 months & $126(0.79)$ & $175(0.80)$ & $28(0.75)$ & 0.94 \\
I2 months & $189(1.18)$ & $27 I(1.24)$ & $39(1.04)$ & 0.57 \\
24 months & $28 I(1.76)$ & $400(1.83)$ & $59(1.57)$ & 0.53 \\
48 months & $388(2.43)$ & $524(2.40)$ & $85(2.27)$ & 0.84 \\
Overall & $450(2.82)$ & $616(2.82)$ & $102(2.72)$ & 0.94 \\
\hline
\end{tabular}

to human error, which may influence the statistical results. Second, we identified sexual dysfunction patients according to ICD-9-CM codes (302 and 607). Patients with sexual problems visited the outpatient department for consultation, but we could not determine whether these patients had ED, retrograde dysfunction, or psychological problems. Third, we could only check the diagnosis of BPH, and we did not have prostate echography results for analysis of the prostate size.

\section{Conclusion}

Among Taiwanese patients undergoing TURP, the resected prostate weight might not be related with the occurrence or onset time of postoperative sexual dysfunction after adjusting for age, chronic renal failure, ischemic heart disease, and obesity.

\section{Acknowledgments}

This study was based on data from the NHI research database provided by the Bureau of National Health Insurance, Department of Health and managed by the National Health Research Institutes. The interpretations and conclusions contained herein do not represent the views of the Bureau of National Health Insurance, Department of Health, or National Health Research Institutes.

\section{Author contributions}

Conceptualization: F-CL, S-JS, and H-PY. Data acquisition: J-RL. Formal analysis: F-CL, S-JS, and J-RL. Funding acquisition: H-PY. Investigation: F-CL, S-JS, and H-PY. Methodology: J-RL. Project administration: F-CL, S-JS, and H-PY. Resources: F-CL and H-PY. Supervision: H-PY. Writing draft: F-CL, S-JS, and H-PY. Critical review and editing: all authors. All authors contributed to data analysis, drafting and revising the article, gave final approval of the version to be published, and agree to be accountable for all aspects of the work. 


\section{Disclosure}

The authors report no conflicts of interest in this work.

\section{References}

1. Marra G, Sturch P, Oderda M, Tabatabaei S, Muir G, Gontero P. Systematic review of lower urinary tract symptoms/benign prostatic hyperplasia surgical treatments on men's ejaculatory function: time for a bespoke approach? Int J Urol. 2016;23(1):22-35.

2. Rosen R, Altwein J, Boyle P, et al. Lower urinary tract symptoms and male sexual dysfunction: the multinational survey of the aging male (MSAM-7). Eur Urol. 2003;44(6):637-649.

3. Bell JR, Laborde E. Update on the sexual impact of treatment for benign prostatic hyperplasia. Curr Urol Rep. 2012;13(6):433-440.

4. Pavone C, Abbadessa D, Scaduto G, et al. Sexual dysfunctions after transurethral resection of the prostate (TURP): evidence from a retrospective study on 264 patients. Arch Ital Urol Androl. 2015;87(1):8-13.

5. Gacci M, Eardley I, Giuliano F, et al. Critical analysis of the relationship between sexual dysfunctions and lower urinary tract symptoms due to benign prostatic hyperplasia. Eur Urol. 2011;60(4):809-825.

6. Li Z, Chen P, Wang J, et al. The impact of surgical treatments for lower urinary tract symptoms/benign prostatic hyperplasia on male erectile function: a systematic review and network meta-analysis. Medicine (Baltimore). 2016;95(24):e3862.

7. Jaidane M, Arfa NB, Hmida W, et al. Effect of transurethral resection of the prostate on erectile function: a prospective comparative study. Int $J$ Impot Res. 2010;22(2):146-151.

8. Reich O, Gratzke C, Stief CG. Techniques and long-term results of surgical procedures for BPH. Eur Urol. 2006;49(6):970-978.

9. Becher EF, McVary KT. Surgical procedures for BPH/LUTS: impact on male sexual health. Sex Med Rev. 2014;2(1):47-55.
10. Zong HT, Peng XX, Yang CC, Zhang Y. The impact of transurethral procedures for benign prostate hyperplasia on male sexual function: a meta-analysis. $J$ Androl. 2012;33(3):427-434.

11. Mamoulakis C, Skolarikos A, Schulze M, et al. Bipolar vs monopolar transurethral resection of the prostate: evaluation of the impact on overall sexual function in an international randomized controlled trial setting. BJU Int. 2013;112(1):109-120.

12. McVary KT, McKenna KE. The relationship between erectile dysfunction and lower urinary tract symptoms: epidemiological, clinical, and basic science evidence. Curr Urol Rep. 2004;5(4):251-257.

13. Tarcan T, Azadzoi KM, Siroky MB, Goldstein I, Krane RJ. Age-related erectile and voiding dysfunction: the role of arterial insufficiency. Br J Urol. 1998;82(Suppl 1):26-33.

14. Gandhi J, Weissbart SJ, Smith NL, et al. The impact and management of sexual dysfunction secondary to pharmacological therapy of benign prostatic hyperplasia. Transl Androl Urol. 2017;6(2):295-304.

15. Oelke M, Bachmann A, Descazeaud A, et al. EAU guidelines on the treatment and follow-up of non-neurogenic male lower urinary tract symptoms including benign prostatic obstruction. Eur Urol. 2013;64(1): 118-140.

16. Briganti A, Naspro R, Gallina A, et al. Impact on sexual function of holmium laser enucleation versus transurethral resection of the prostate: results of a prospective, 2-center, randomized trial. J Urol. 2006;175(5): $1817-1821$.

17. Tscholl R, Largo M, Poppinghaus E, Recker F, Subotic B. Incidence of erectile impotence secondary to transurethral resection of benign prostatic hyperplasia, assessed by preoperative and postoperative Snap Gauge tests. J Urol. 1995;153(5):1491-1493.

18. Favilla V, Cimino S, Salamone C, et al. Risk factors of sexual dysfunction after transurethral resection of the prostate (TURP): a 12 months follow-up. J Endocrinol Invest. 2013;36(11):1094-1098.
Therapeutics and Clinical Risk Management

\section{Publish your work in this journal}

Therapeutics and Clinical Risk Management is an international, peerreviewed journal of clinical therapeutics and risk management, focusing on concise rapid reporting of clinical studies in all therapeutic areas, outcomes, safety, and programs for the effective, safe, and sustained use of medicines. This journal is indexed on PubMed Central, CAS,

\section{Dovepress}

EMBase, Scopus and the Elsevier Bibliographic databases. The manuscript management system is completely online and includes a very quick and fair peer-review system, which is all easy to use. Visit http://www.dovepress.com/testimonials.php to read real quotes from published authors. 